\title{
Radiation Patterns of Seismic Surface Waves from Buried Dipolar Point Sources in a Flat Stratified Earth ${ }^{1}$
}

\author{
Ari Ben-Menahem and David G. Harkrider \\ Seismological Laboratory \\ California Institute of Technology, Pasadena
}

\begin{abstract}
Explicit compact expressions were obtained for the far displacement field of Rayleigh and Love waves generated by force configurations which served to simulate sheartype faults with arbitrary dip and slip. The medium transfer functions for dipolar sources were computed for a Gutenberg flat continental earth model with 23 layers. These were then used to obtain universal radiation pattern charts for couple- and double-couple-type sources at various depths over the period range 50 to 350 sec. It was demonstrated by means of few typical examples that the radiation patterns of Rayleigh waves may depend strongly on the depth of the source, and unlike the fundamental Love mode may be rather sensitive to small variations in frequency. For a given source and frequency the radiation pattern may differ considerably from one mode to another.
\end{abstract}

\section{INTRODUCTION}

The inverse problem of reconstructing the seismic source from amplitude measurements at stations encircling the epicenter was hitherto mainly restricted to first-motion studies. In spite of the fact that certain amounts of information could be obtained from a tiny portion of the seismogram, seismologists soon realized that the complexity of earthquake origins in space and time could not be adequately understood and uniquely determined unless ways and means were found to extract the source parameters from the main body of the seismogram.

The advent of long-period seismographs and high-speed computers opened new vistas in this field. Analysis of surface waves and free oscillations improved our knowledge and supplied us with additional tools for future research.

The present availability of long-period records from the archives of the USCGS worldwide net of standardized stations will undoubtedly put source mechanism studies on a new level. To prepare the grounds for studies of this kind, it is necessary to know first the theoretical radiation pattern of surface waves from various sources. Yanovskaya [1958] calculated the response of a layer over a half-space to Love and Rayleigh waves due to singlets and couples. Ben-Menahem [1961] calculated radiation pat-

1 Contribution 1237, Division of Geological Sciences, California Institute of Technology, Pasadena. terns for Rayleigh waves in a half-space due to moving faults. Harkrider [1964b] calculated the response of a multilayered half-space to Love and Rayleigh waves due to buried horizontal and vertical point forces. Haskell [1963] calculated radiation patterns for Rayleigh waves generated by dipolar point sources at depth in a homogeneous isotropic half-space. The present paper supplies the general theory of Rayleigh and Love wave radiation from dipolar point sources with arbitrary elements in a multilayered earth. The theory is then applied to a continental Gutenberg earth model.

\section{List of Symbols}

$a_{0}$ Coefficient in the expression for the reduced displacements (Table 3).

$a_{R_{m}}, a_{L_{m}}$ Rayleigh and Love matrices of the $m$ th layer.

$A_{R_{\mathrm{m}}}, A_{L_{m}}$ The Rayleigh and Love product matrices of $m$ layers.

$A_{R}, A_{L}$ The Rayleigh and Love amplitude factors.

$A(h)$ Rayleigh wave ellipticity function.

$b_{0}, b_{1}$ Coefficients in the expression for the reduced displacements (Table 3 ).

$B(h, \omega)$ Rayleigh wave first dipolar transfer function.

$c(\omega)$ Phase velocity.

$c_{R}, c_{L}$ Rayleigh and Love phase velocities.

$C(h, \omega)$ Rayleigh wave second dipolar transfer function. 
$d_{i}$ Radiation pattern coefficients (Table 1).

$\mathbf{e}_{1}, \mathbf{e}_{2}, \mathbf{e}_{3}$ Unit vectors at the source along the strike, dip, and the vertical directions respectively.

$\mathbf{e}_{r}, \mathbf{e}_{\theta}, \mathbf{e}_{\mathbf{z}}$ Unit vectors along the radial, azimuthal and vertical axes at the recording station.

$\left(\mathbf{F}_{r}, \mathbf{F}_{\theta}, \mathbf{F}_{z}\right)$ A three vector representation of the total far-displacement field at the recording station.

$G(h, \omega)$ Love wave dipolar transfer function.

$h$ Source depth.

$H_{0}{ }^{(2)}\left(k_{n} r\right), H_{1}{ }^{(2)}\left(k_{n} r\right)$ Hankel functions.

$k_{n}$ Wave number of $n$th mode.

$k_{R}, k_{L}$ Rayleigh and Love wave numbers.

$L$ Conversion factor.

$m_{R}(\theta), m_{L}(\theta)$ Rayleigh and Love radiation pattern amplitudes.

M Moment of couple.

n Normal vector to plane of motion.

$N_{\theta}(h, \omega)$ Love wave singlet transfer function.

$N_{r r}(h, \omega)$ Rayleigh wave first singlet transfer function.

$N_{r s}(h, \omega)$ Rayleigh wave second singlet transfer function.

$\mathbf{R}(\omega)$ The time transformed displacement vector in plane of motion.

$t$ Time.

$T$ Period of a spectral component.

$\frac{\dot{u}_{S}(h)}{\dot{w}_{0}}, \frac{\dot{v}_{\mathcal{S}}(h)}{\dot{v}_{0}}, \frac{\dot{w}_{S}(h)}{\dot{w}_{0}}$ Haskell's plane wave par-

ticle velocity (or displacement) ratios.

$\dot{u}_{0}^{*}=-i \dot{u}_{0}$.

$U$ Group velocity.

$\mathrm{U}(\omega)$ The time transformed vector displacement field.

$\overline{\mathbf{U}}(\omega)$ Complex conjugate of $\mathrm{U}(\omega)$.

$\mathrm{U}^{s}, \mathbf{U}^{c}, \mathrm{U}^{D C}$ The time transformed vector displacement fields due to singlet, couple, and double-couple sources, respectively.

$U_{r}, U_{\theta}, U$, Components of time transformed displacement field in cylindrical coordinates.

$|U(r, \theta, \omega)|$ Spectral amplitude of ground motion at the recording station.

$U^{*}(r, \theta, \omega)$ The reduced displacement.

$\left|U^{*}(r, \theta, \omega)\right|$ The reduced amplitude.

$U_{0}$ Coefficient in the expression for the reduced displacements (Table 3).

$z$ Depth of receiver (station).

$\alpha$ Compressional velocity.

$\alpha_{0}$ Compressional velocity at depth $h=0$.

$\alpha_{s}$ Compressional velocity in the source layer. $\beta$ Shear velocity. $\beta_{0}$ Shear velocity at depth $h=0$.

$\beta_{S}$ Shear velocity in the source layer.

$\gamma(\omega)$ Absorption coefficient function for surface waves.

$\epsilon_{0}$ Rayleigh wave surface ellipticity.

$\mu$ Rigidity.

$\mu_{S}$ Rigidity of the source layer.

$\rho$ Density.

$\sigma_{R \mathrm{~s}}(h)$ Depth-dependent factor of normal stress associated with the Rayleigh waves.

$\sigma_{0}$ Poisson ratio at depth $h=0$.

$\tau_{R_{S}}(h), \tau_{L_{S}}(h)$ Depth-dependent factors of tangential stresses associated with the Rayleigh and Love waves.

$\phi_{R}, \phi_{L}$ Rayleigh and Love spectral spatial phases. $\chi(\theta)$ The radiation pattern function.

$\omega$ Angular frequency.

Other symbols defined in Figure 1.

\section{Coordinate Ststem, Notation, and Sign Convention}

We consider a shear-type fault with arbitrary dip and slip in a layered earth. We replace the physical fault by an equivalent force system. This force system is generated from a single force, which is directed along the fault's motion and has a magnitude proportional to the amount of displacement in the plane of motion. We can furthermore assume, without loss of generality, that the time dependence of the force is that of the Dirac delta function.

The geometry of the situation is displayed in Figure 1. Following Haskell [1963] we choose the source coordinate system to be cartesian with the $x_{1}$ axis in the strike direction, the $x_{2}$ axis in the dip direction, and the $x_{3}$ axis in the upward vertical direction. The convention is followed that all three force components are positive on the hanging-wall side of a reverse left-lateral fault. Hence the vector $\mathbf{R}(\omega)$ will be represented by

$$
\begin{aligned}
\mathbf{R}= & |R|\left\{\cos \lambda \mathbf{e}_{1}\right. \\
& \left.+\sin \lambda \cos \delta \mathrm{e}_{2}+\sin \lambda \sin \delta \mathbf{e}_{3}\right\}
\end{aligned}
$$

Likewise, the normal is given by

$$
\mathbf{n}=|n|\left\{-\sin \delta \mathrm{e}_{2}+\cos \delta \mathrm{e}_{3}\right\}
$$

where $0 \leq \delta \leq \pi$ and $0 \leq \lambda \leq 2 \pi$.

\section{Single Force in a Multilayered Medidm}

It has been shown by Ben-Menahem and Toksöz [1963] that the displacement field of an arbitrary 


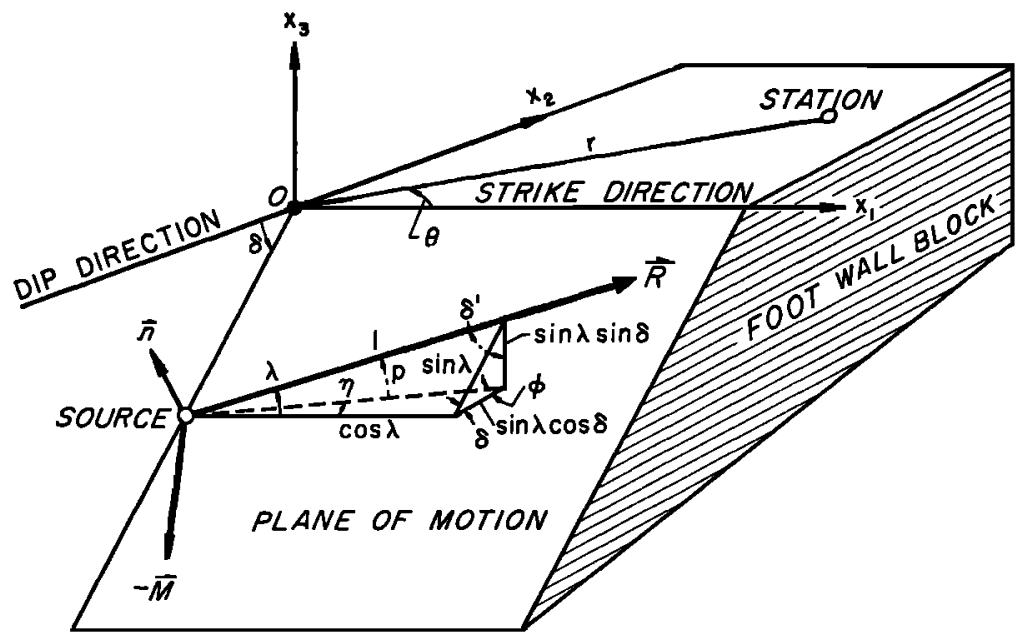

Fig. 1. Geometry of source elements and relative position of station on the free surface.

force system in a multilayered medium can be generated from the response of the medium to three mutual perpendicular unit forces.

The position of the recording station is given by $(\theta, r, z=0)$ in the source cylindrical system (see Figure 1). $\theta$ is measured positively anticlockwise from the positive strike direction $\left(x_{1}=0\right)$ when viewed from above the half-space downward. $r$ is measured positively outward from the source. Thus we can write the fardisplacement field $\mathbf{U}^{s}$ due to a single force $\mathbf{R}$ at the source as

$\mathbf{U}^{S}=\left(\mathbf{R} \cdot \mathbf{F}_{\theta}\right) \mathbf{e}_{\theta}+\left(\mathbf{R} \cdot \mathbf{F}_{r}\right) \mathbf{e}_{r}+\left(\mathbf{R} \cdot \mathbf{F}_{z}\right) \mathbf{e}_{z}$

where

$$
\begin{aligned}
& \mathbf{F}_{\theta}=\left(\sin \theta e^{i \pi \pi / 4} N_{\theta}, \cos \theta e^{-i \pi / 4} N_{\theta}, 0\right) \\
& \mathbf{F}_{r}=\left(\cos \theta e^{-i \pi / 4} N_{r r},\right. \\
&\left.\quad \sin \theta e^{-i \pi / 4} N_{r r}, e^{i \pi / 4} N_{r z}\right) \\
& \mathbf{F}_{z}=\left(\cos \theta e^{-i 3 \pi / 4} N_{z r},\right. \\
&\left.\sin \theta e^{-i 3 \pi / 4} N_{z r}, e^{-i \pi / 4} N_{z z}\right)
\end{aligned}
$$

Using the representation of $\mathbf{R}$ as given in (1) we obtain the far-displacement field in terms of the elements of the fault $(\lambda, \delta)$ and the azimuth angle $\theta$.

$$
\begin{gathered}
U_{\theta}{ }^{S}=|R| N_{\theta} e^{i 3 \pi / 4}(\sin \theta \cos \lambda \\
-\cos \theta \sin \lambda \cos \delta) \\
U_{r}{ }^{s}=|R| e^{-i \pi / 4}\left[N_{r r}(\cos \theta \cos \lambda\right.
\end{gathered}
$$

$$
\left.+\sin \theta \sin \lambda \cos \delta)+i N_{r z} \sin \lambda \sin \delta\right]
$$

$$
U_{x} / U_{z}=\epsilon_{0} e^{i \pi / 2}
$$

In these expressions the factor $(2 \pi r)^{-1 / 2} e^{i\left(\omega t-k_{n} r\right)}$ has been suppressed. All other constants are incorporated in the definition of $N_{\theta}, N_{r r}$, and $N_{r z}$. The symbol $N_{i i}$ which appears in (8) and (9) stands for the component of the amplitude response of the medium in the $i$ direction (horizontal or vertical) due to a unit force acting along the $j$ direction (horizontal or vertical). Thus, for example, we write $N_{r z}$ for the horizontal response due to a vertical force. These functions are obtained directly from the exact solution to the boundary value problem. They depend on the frequency, the depth of the source, and the constants of the layered medium. $N_{\theta}$ is the Love wave response of the medium to a horizontal force. The factor $\left(k_{n}\right)^{-1 / 2}$ which arises from the asymptotic expansion approximation of $H_{0,1}{ }^{(2)}\left(k_{n} r\right)$ is absorbed in $N_{i i}$.

Comparing equations 3 to 9 with the solutions given by Harkrider [1964a] for vertical and horizontal force at depth in a multilayered halfspace, we can express the functions $N_{i j}$ at $z=0$ as

$$
\begin{gathered}
N_{z r}(h)=-\left[\dot{u}_{S}^{*}(h) / \dot{w}_{0}\right] \mathrm{A}_{R} k_{R}^{-1 / 2} \\
N_{r r}(h)=-\left[\dot{u}_{0}^{*} / \dot{w}_{0}\right] N_{z r}(h)=\epsilon_{0} N_{z r}(h) \\
N_{z z}(h)=\left[\dot{w}_{S}(h) / \dot{w}_{0}\right] \mathrm{A}_{R} k_{R}{ }^{-1 / 2} \\
N_{r s}(h)=\epsilon_{0} N_{s z}(h)
\end{gathered}
$$




$$
N_{\theta}(h)=\left[\dot{v}_{S}(h) / \dot{v}_{0}\right] \mathrm{A}_{L} k_{L}^{-1 / 2}
$$

The amplitude factors $A_{L}$ and $A_{R}$ are functions of frequency and the elastic properties of the multilayered array. They are independent of the source type and depth. This is also true for the ellipticity. The Rayleigh wave amplitude factor $A_{R}$ also occurs in the solution to an explosive source at depth. The properties of $A_{R}$ and $A_{L}$ are given in more detail by Harkrider [1964a, b].

The depth-dependent factor in equations 10 to 14 can be represented in terms of the ThomsonHaskell layer matrices [Haskell, 1953] as

$$
\begin{aligned}
& {\left[\dot{u}_{s} *(h) / \dot{w}_{0}\right]=\left[A_{R s}(h)\right]_{12} *-\epsilon_{0}\left[A_{R s}(h)\right]_{11}} \\
& {\left[\dot{w}_{S}(h) / \dot{w}_{0}\right]=\left[A_{R_{S}}(h)\right]_{22}+\epsilon_{0}\left[A_{R S}(h)\right]_{21} *} \\
& {\left[\dot{v}_{S}(h) / \dot{v}_{0}\right]=\left[A_{L s}(h)\right]_{11}} \\
& A_{R s}(h)=\mathbb{Q}_{R_{s}}(h) \mathfrak{Q}_{R s-1} \cdots \mathfrak{Q}_{R 1} \\
& A_{L_{s}}(h)=\mathfrak{Q}_{L_{s}}(h) \mathfrak{Q}_{L_{s-2}} \cdots \mathfrak{Q}_{L_{1}}
\end{aligned}
$$

and $a_{R_{m}}, a_{L_{m}}$ are the Rayleigh and Love matrices of the $m$ th layer. The source layer is indicated by $m=S$ and the surface layer by $m=1$. The matrices $a_{R_{B}}(h)$ and $a_{L_{B}}(h)$ are layer matrices for a sublayer in the source layer, the thickness of which is equal to the penetration of the source depth $h$ into the source layer. From equations 10 to 14 we see that

$\epsilon_{0}=N_{r z}(h) / N_{z z}(h)=N_{r r}(h) / N_{z r}(h)$

for all source depths. Since, by definition, $\dot{u}_{0} \equiv \dot{u}_{1}(0)$ and $\dot{w}_{0} \equiv \dot{w}_{1}(0)$, it follows that for a surface source, $h=0$,

$$
N_{r z}(0)=N_{z r}(0)
$$

\section{Generation of Dipolar Sodrce}

The couple displacements are obtained by the application of a differential operator to the displacement vector $\mathrm{U}^{s}$ due to a single force. Thus

$$
\begin{gathered}
\mathbf{U}^{c}=-(\mathbf{n} \cdot \operatorname{grad}) \mathbf{U}^{s}=\left(\sin \theta \sin \delta \frac{\partial}{\partial r}\right. \\
\left.-\cos \delta \frac{\partial}{\partial h}\right) \mathbf{U}^{s}+O\left(r^{-3 / 2}\right)
\end{gathered}
$$

We must choose here the negative sign for the gradient because the derivative is taken at the source, the station remaining fixed. Since we are interested only in the far field, we have discarded terms of higher order than $r^{-1 / 2}$. The vector $n$ is a unit vector with dimensions of length.

The double-couple source is obtained by the superposition of two equal couples at right angles so that the total moment of the system is zero. To obtain the far-displacement field for this system we add to the operational representation of $\mathrm{U}^{c}$ as given by (22) the displacement field due to a second couple which is formed by the interchange of $\mathbf{R}$ with $\mathbf{n}$. Hence

$$
\begin{aligned}
\mathrm{U}^{D C}=-(\mathbf{n} \cdot \operatorname{grad}) \mathbf{U}^{\mathcal{S}}(\mathbf{R}) \\
-\left(\frac{\mathbf{R}}{|R|} \cdot \operatorname{grad}\right) \mathbf{U}^{S}(|R| \mathbf{n})
\end{aligned}
$$

Performing the operations indicated by (23) we obtain general formulas for the far field of Love and Rayleigh waves. The general form of this field may be written as

$$
\mathrm{U}=|R||n| k_{n} e^{-i 3 \pi / 4} N(h) \chi(\theta)
$$

where $k_{n}$ is either $k_{R}$ or $k_{L}, N(h)$ is either $N_{\theta}(h)$ or $N_{r q}(h)$, and $\chi(\theta)$ is the complex function

$$
\begin{aligned}
\chi(\theta)=d_{0} & +i\left(d_{1} \sin \theta+d_{2} \cos \theta\right) \\
& +d_{3} \sin 2 \theta+d_{4} \cos 2 \theta
\end{aligned}
$$

The coefficients $d_{i}$ are given in Table $1 . \lambda$ is measured counterclockwise from the positive strike direction, and $\delta$ is measured downward from the negative dip direction. The dimensionless entities $A, B, C$, and $G$ are given by

$$
\begin{gathered}
A(h)=N_{r r}(h) / N_{r z}(h) \\
B(h)=\left(N_{r r}(h)+\frac{2}{k_{R}} \frac{\partial N_{r z}(h)}{\partial h}\right) / N_{r z}(h) \\
C(h)=\left[N_{r z}(h)+\frac{1}{k_{R}} \frac{\partial N_{r r}(h)}{\partial h}\right] / N_{r z}(h) \\
G(h)=\frac{\partial N_{\theta}(h)}{\partial h} / k_{L} N_{\theta}(h)
\end{gathered}
$$

The common phase constant $e^{-i a r / 4}$, appearing in (24) has been chosen so as to render the functions $A, B, C$, and $G$ non-negative at $h=0$. The signs in these functions are adjusted in such a way that $h$ is increasing positively downward. The complex function $\chi(\theta)$ defines two real functions of the real variable $\theta$. Its modulus $|\chi(\theta)|$ gives the amplitude radiation pattern, and its argument arg $\chi(\theta)$ gives the spatial phase [Ben-Menahem and Toksöz, 1963] of the source. 
TABLE 1. Radiation Pattern Coefficients

\begin{tabular}{|c|c|c|c|c|}
\hline \multirow[b]{2}{*}{ Coefficient } & \multicolumn{2}{|c|}{ Couple } & \multicolumn{2}{|c|}{ Double Couple } \\
\hline & Love & Rayleigh & Love & Rayleigh \\
\hline$d_{0}$ & $-1 / 2 \cos \lambda \sin \delta$ & $1 / 4 \sin \lambda \sin 2 \delta B(h)$ & $\mathbf{0}$ & $1 / 2 \sin \lambda \sin 2 \delta B(h)$ \\
\hline$d_{1}$ & $\cos \lambda \cos \delta G(h)$ & $\sin \lambda\left[1-C(h) \cos ^{2} \delta\right]$ & $\cos \lambda \cos \delta G(h)$ & $-\sin \lambda \cos 2 \delta C(h)$ \\
\hline$d_{2}$ & $-\sin \lambda \cos ^{2} \delta G(h)$ & $\cos \lambda \cos \delta[1-C(h)]$ & $-\sin \lambda \cos 2 \delta G(h)$ & $-\cos \lambda \cos \delta C(h)$ \\
\hline$d_{\mathbf{a}}$ & $1 / 4 \sin \lambda \sin 2 \delta$ & $1 / 2 \cos \lambda \sin \delta A(h)$ & $1 / 2 \sin \lambda \sin 2 \delta$ & $\cos \lambda \sin \delta A(h)$ \\
\hline$d_{4}$ & $1 / 2 \cos \lambda \sin \delta$ & $-1 / 4 \sin \lambda \sin 2 \delta A(h)$ & $\cos \lambda \sin \delta$ & $-1 / 2 \sin \lambda \sin 2 \delta A(h)$ \\
\hline
\end{tabular}

From equations 10 to 19 and the definition of the elements of the layer matrices [Haskell, 1953], the derivatives appearing in the expressions $B(h), C(h)$, and $G(h)$ can be given as

$$
\begin{aligned}
& G(h)=-\frac{1}{\mu_{S}}\left\{\left[A_{L S}(h)\right]_{21} *\right\} /\left[\frac{\dot{v}_{S}(h)}{\dot{v}_{0}}\right] \\
& A(h)=-\left[\dot{u}_{S}{ }^{*}(h) / \dot{w}(h)\right] \\
& B(h)=-\left(3-4 \frac{\beta_{S}{ }^{2}}{\alpha_{S}{ }^{2}}\right)\left[\frac{\dot{u}_{S}{ }^{*}(h)}{\dot{w}_{S}(h)}\right]-\frac{2 \beta_{S}{ }^{2}}{\mu_{S} \alpha_{S}{ }^{2}} \\
& \cdot\left[\frac{\dot{w}_{0}}{\dot{w}_{S}(h)}\right]\left\{\left[A_{R S}(h)\right]_{32}{ }^{*}-\epsilon_{0}\left[A_{R S}(h)\right]_{31}\right\} \\
& C(h)=-\frac{\left[A_{R S}(h)\right]_{42}+\epsilon_{0}\left[A_{R S}(h)\right]_{41} *}{\mu_{S}\left[\frac{\dot{w}_{S}(h)}{\dot{w}_{0}}\right]}
\end{aligned}
$$

where the functions inside the braces have the physical meaning of stress ratios at depth $h$ :

$$
\begin{gathered}
{\left[A_{L_{s}}(h)\right]_{21} *=\left[\frac{\tau_{L_{s}} *(h)}{\dot{v}_{0} / c_{L}}\right]} \\
{\left[A_{R s}(h)\right]_{32} *-\epsilon_{0}\left[A_{R s}(h)\right]_{\mathrm{s} 1}=\left[\frac{\sigma_{R s} *(h)}{\dot{w}_{0} / c_{R}}\right]} \\
{\left[A_{R s}(h)\right]_{42}+\epsilon_{0}\left[A_{R s}(h)\right]_{42} *=\left[\frac{\tau_{R s}(h)}{\dot{w}_{0} / c_{R}}\right]}
\end{gathered}
$$

It can be shown from (31) that $A(h)$ is the ellipticity that a receiver at depth $z=h$ would measure for all source depths.

$$
A(h)=\epsilon(z=h) \quad A(0)=\epsilon(0)=\epsilon_{0}
$$

Since the ellipticity is independent of the source depth, we can write

$$
\epsilon_{0}=\frac{\partial N_{r r}(h) / \partial h}{\partial N_{z r}(h) / \partial h}=\frac{\partial N_{r \varepsilon}(h) / \partial h}{\partial N_{z \varepsilon}(h) / \partial h}
$$

For a surface source, $h=0$, the stress ratios defined by equations 34 to 36 vanish, and we obtain

$$
\begin{aligned}
& B(0)=\epsilon_{0}\left(3-4 \frac{\beta_{0}{ }^{2}}{\alpha_{0}{ }^{2}}\right)=\epsilon_{0}\left(\frac{1+\sigma_{0}}{1-\sigma_{0}}\right) \\
& C(0)=G(0) \equiv 0
\end{aligned}
$$

\section{Character of the Transfer Fonctions of THE MedidM}

Values of the exact expressions for $N_{\theta}, N_{r s}$, $N_{r r}, A, B, C$, and $G$ were computed for a realistic continental earth model. Results were obtained for the fundamental Rayleigh mode and for the fundamental and first Love modes over the period range 50 to $450 \mathrm{sec}$. Source depths were taken at $5-\mathrm{km}$ intervals between 0 and $40 \mathrm{~km}$ and $33-\mathrm{km}$ intervals between 66 and $627 \mathrm{~km}$, 27 points in all. The input layering constants and the output phase and group velocities are shown in Table 2.

The transfer functions are shown in Figures 2 to 20. Each function has been plotted both as a function of the depth of the source and the frequency. Note the slow variation of $G(h)$ with frequency for sources below the low-velocity channel (Figures 3 and 4). Note also the effect of the $\mathrm{M}$ discontinuity on the profile of $G(h)$ as compared with an analogous variation of the shear velocity function (Figure 5). While $N_{r z}(h, \omega)$ follows the behavior of $N_{\theta}(h, \omega)$ in both variables (compare Figures 2 and 8 and Figures 3 and 9$)$, the function $N_{r r}(h, \omega)$ differs from it considerably, mainly because of its spectral nodes, which depend strongly on the depth of the source (Figures 6 and 7). Another interesting result is the slow variation of $B(h)$ with period for source depths up to $300 \mathrm{~km}$ (Figure 12). 
TABLE 2. Constants of a Gutenberg Flat Continental Earth Model and Calculated Velocities for Three Surface Wave Modes

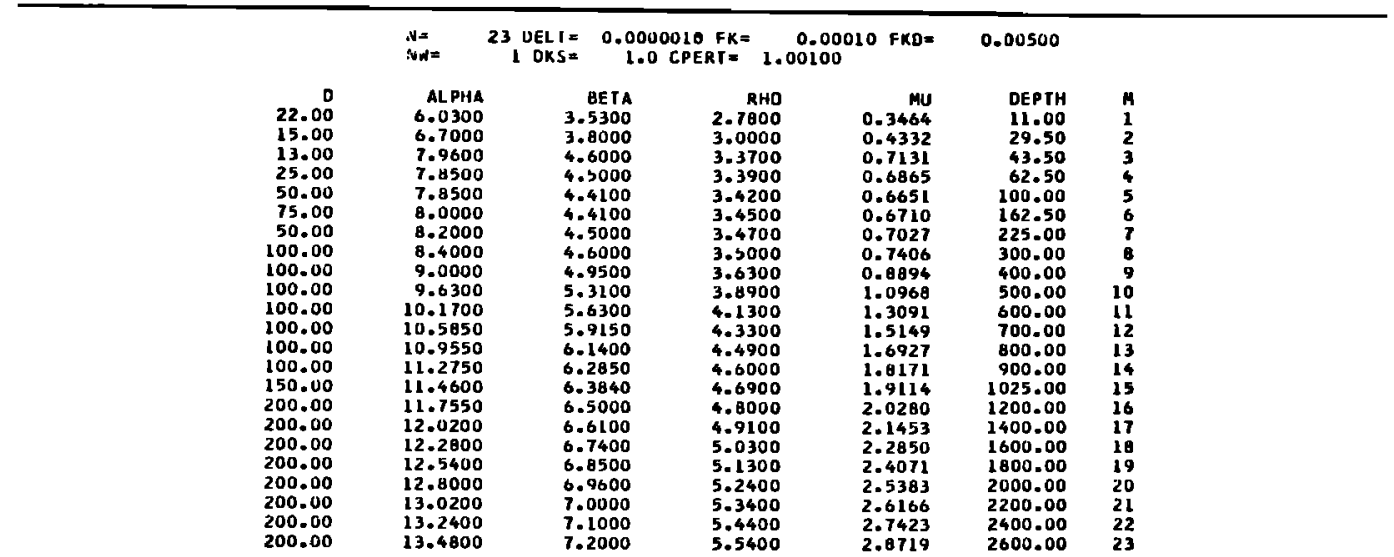

rayleigh wave spectral values for hll

\begin{tabular}{|c|c|c|c|c|}
\hline $\begin{array}{l}.1438 \mathrm{E} \\
.1163 \mathrm{t} \\
.7673 \mathrm{E} \\
.3770 \mathrm{E} \\
.2634 \mathrm{E} \\
.2392 \mathrm{E} \\
.2073 \mathrm{E} \\
.1791 \mathrm{E}\end{array}$ & $\begin{array}{l}03 \\
03 \\
03 \\
03 \\
03 \\
03 \\
03 \\
03 \\
03 \\
03 \\
03\end{array}$ & $\begin{array}{l}0.5600 \mathrm{E} \\
0.3500 \mathrm{E} \\
0.5400 \mathrm{E} \\
0.5300 \mathrm{E} \\
0.3200 \mathrm{E} \\
0.5100 \mathrm{E} \\
0.5000 \mathrm{E} \\
0.4900 \mathrm{E} \\
0.4800 \mathrm{E} \\
0.4700 \mathrm{E} \\
0.4400 \mathrm{E} \\
0.4300 \mathrm{E} \\
0.4200 \mathrm{E} \\
0.4100 \mathrm{E} \\
0.4000 \mathrm{E} \\
0.3900 \mathrm{E} \\
0.3800 \mathrm{E} \\
0.3700 \mathrm{E} \\
0.3600 \mathrm{E} \\
0.3500 \mathrm{E} \\
0.3400 \mathrm{E} \\
0.3300 \mathrm{E}\end{array}$ & $\begin{array}{ll}01 \\
01 \\
01 \\
01 \\
01 \\
01 \\
01 \\
01 \\
01 \\
01 \\
01 \\
01 \\
01 \\
01 \\
01 \\
01 \\
011 \\
01 \\
01 \\
01 \\
01 \\
01 \\
01\end{array}$ & $\begin{array}{l}0 . \\
0 . \\
0 . \\
0 . \\
0 . \\
0 . \\
0 . \\
0 . \\
0 .\end{array}$ \\
\hline
\end{tabular}

love mave spectral values for mode lo

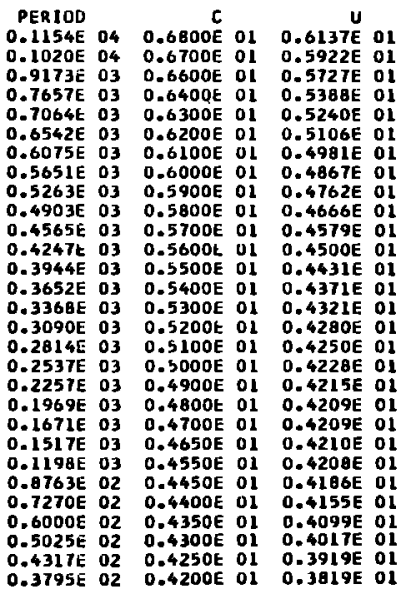

love have spectral values for mode ll

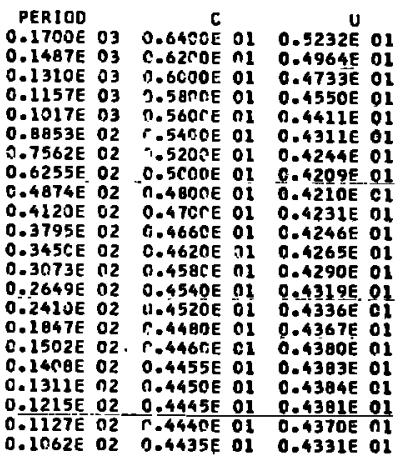

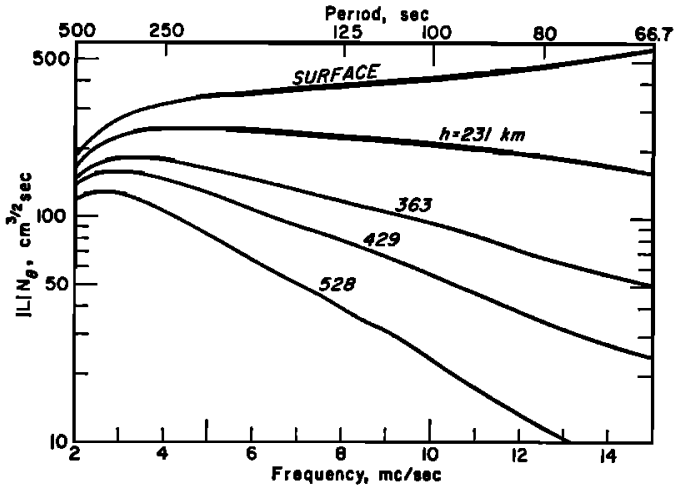

Fig. 2. Spectrum of Love wave singlet transfer function for several values of the source depth. (Here and in the following figures reference is made to the fundamental mode unless otherwise stated.) $L=10^{18.5}$ dyne-sec.

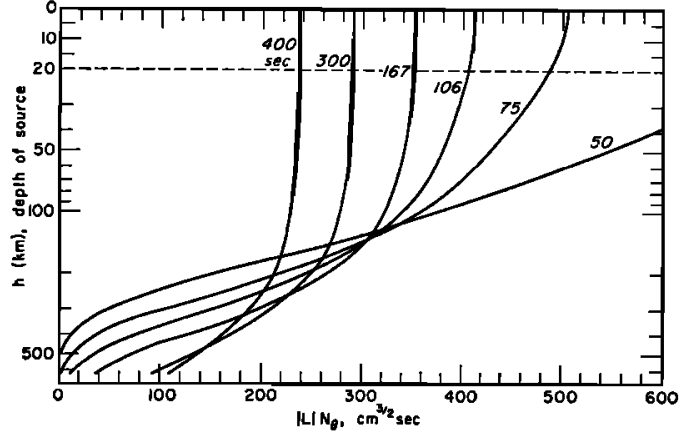

Fig. 3. Variation of Love wave singlet trangfer function with depth of source for various periods. 


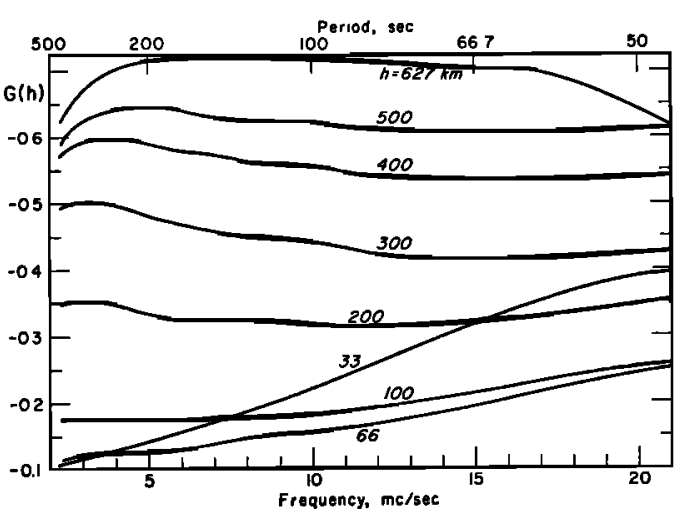

Fig. 4. Spectrum of Love wave dipolar transfer function for several values of the source depth.

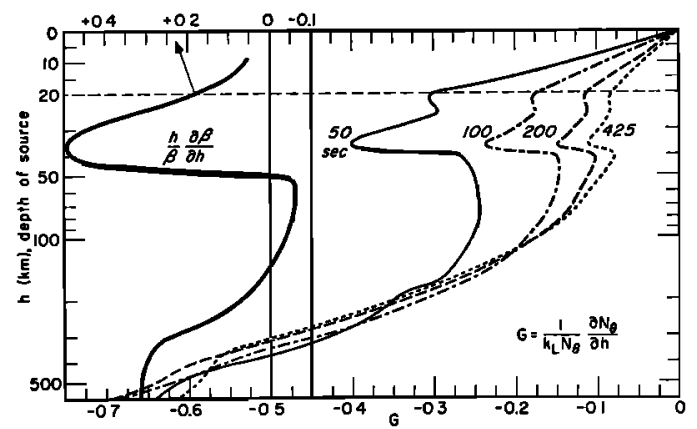

Fig. 5. Variation of Love wave dipolar transfer function with depth of source for various periods. The corresponding variation of the shear wave velocity with depth is also shown.

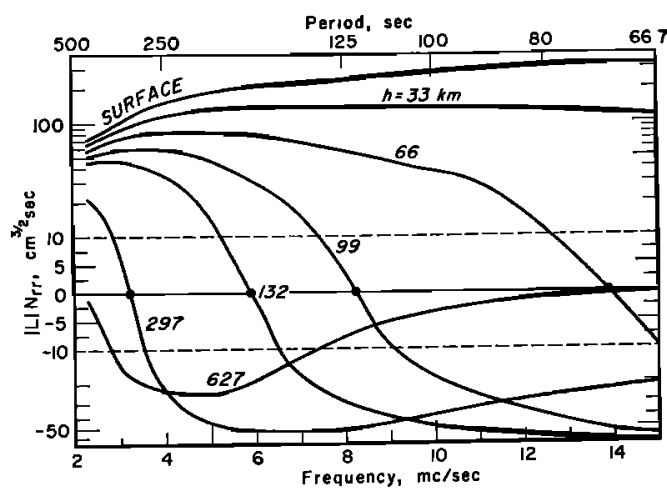

Fig. 6. Spectrum of Rayleigh wave first singlet transfer function for several values of the source depth.

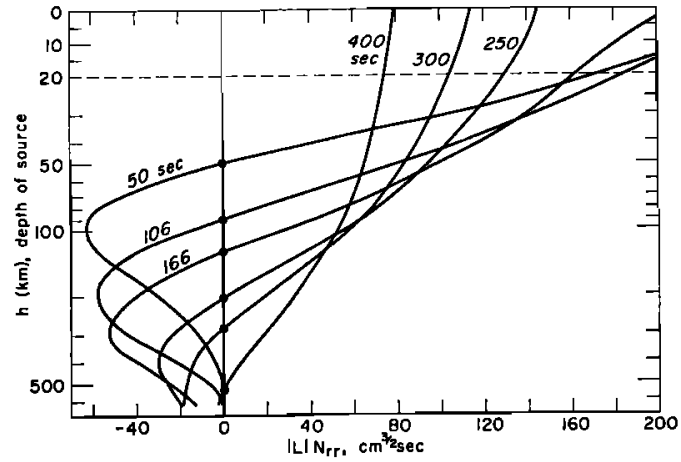

Fig. 7. Variation of Rayleigh wave first singlet transfer function with depth of source for various periods.

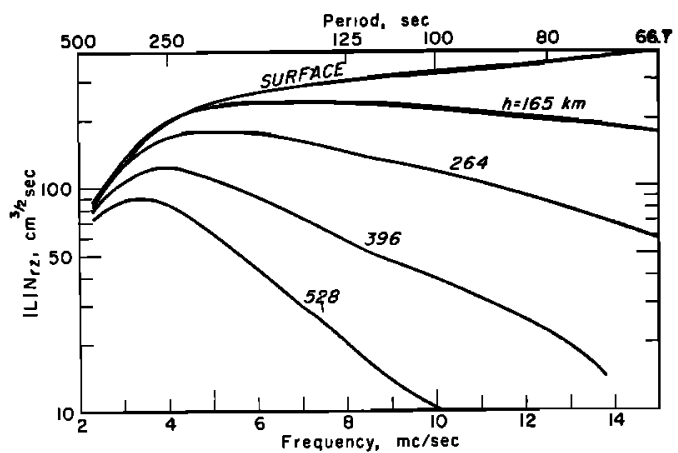

Fig. 8. Spectrum of Rayleigh wave second singlet transfer function for several values of the source depth.

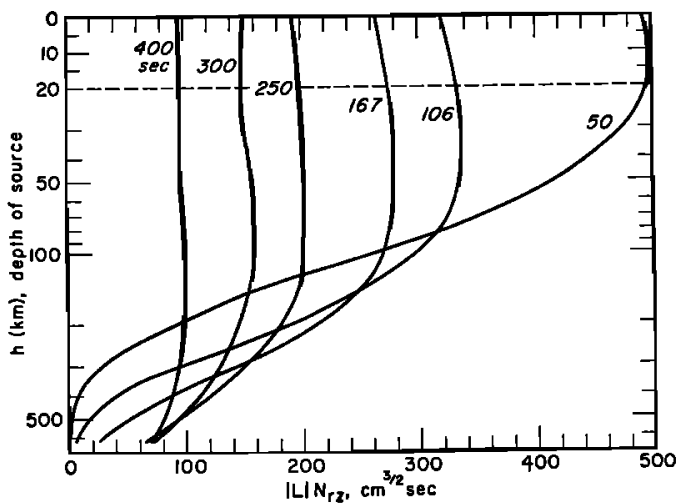

Fig. 9. Variation of Rayleigh wave second singlet transfer function with depth of source for various periods. 


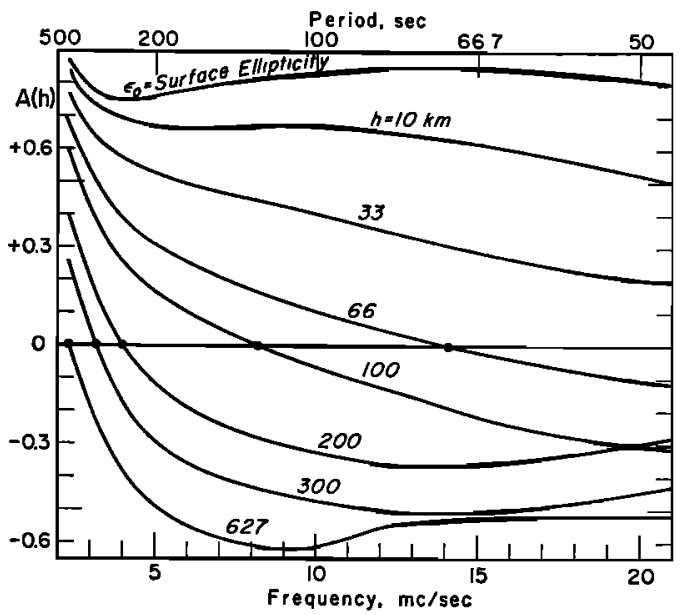

Fig. 10. Spectrum of Rayleigh wave ellipticity function for several depths.

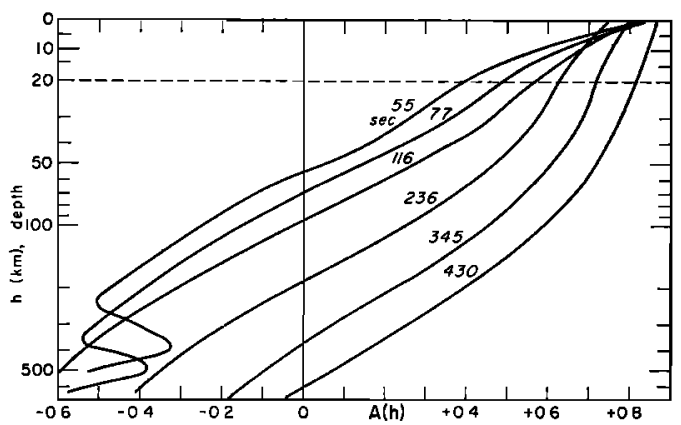

Fig. 11. Variation of Rayleigh wave ellipticity function with depth for various periods.

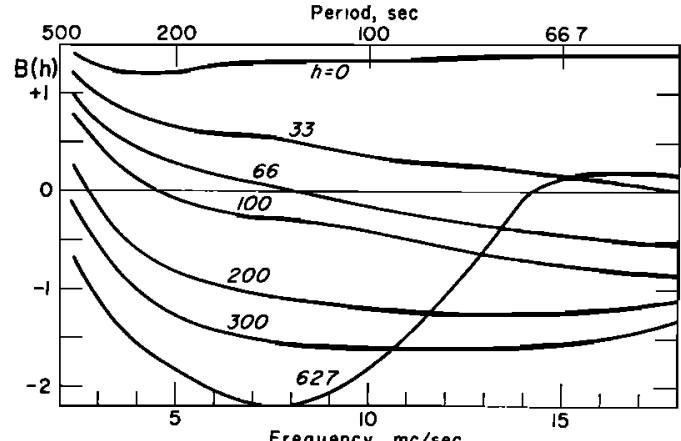

Fig. 12. Spectrum of Rayleigh wave first dipolar function for several values of the source depth.

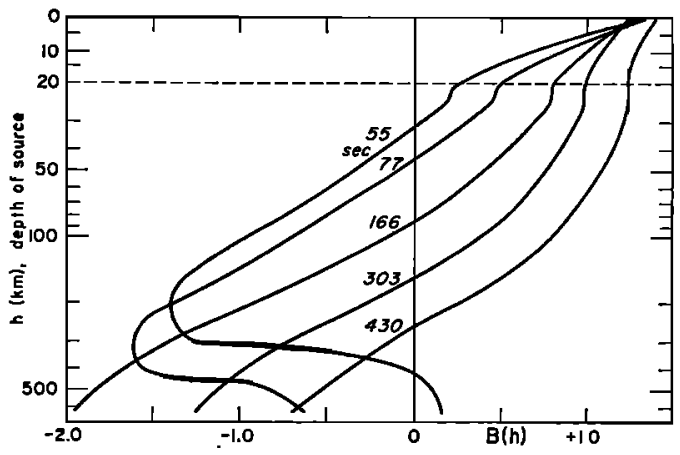

Fig. 13. Variation of the Rayleigh wave first dipolar function with depth of source for various periods.

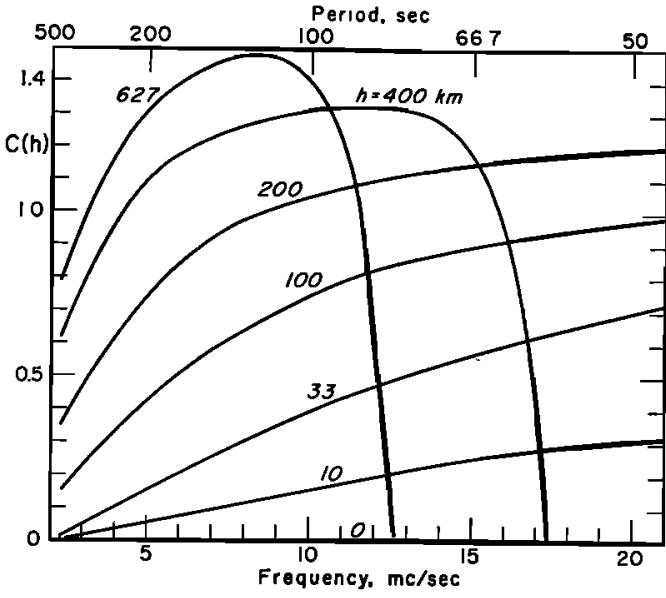

Fig. 14. Spectrum of Rayleigh wave second dipolar function for several values of the source depth.

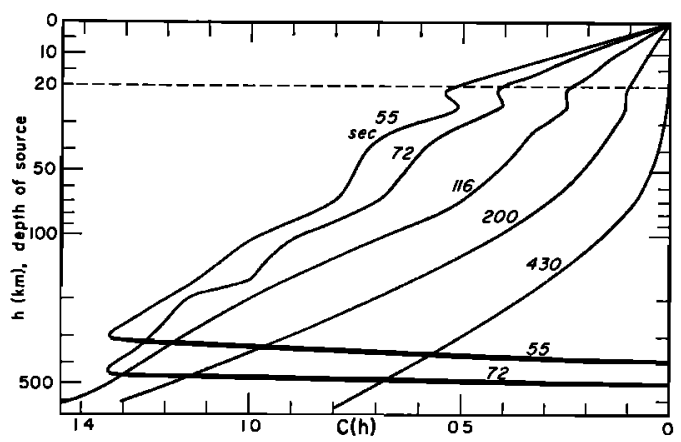

Fig. 15. Variation of Rayleigh wave second dipolar function for various periods. 

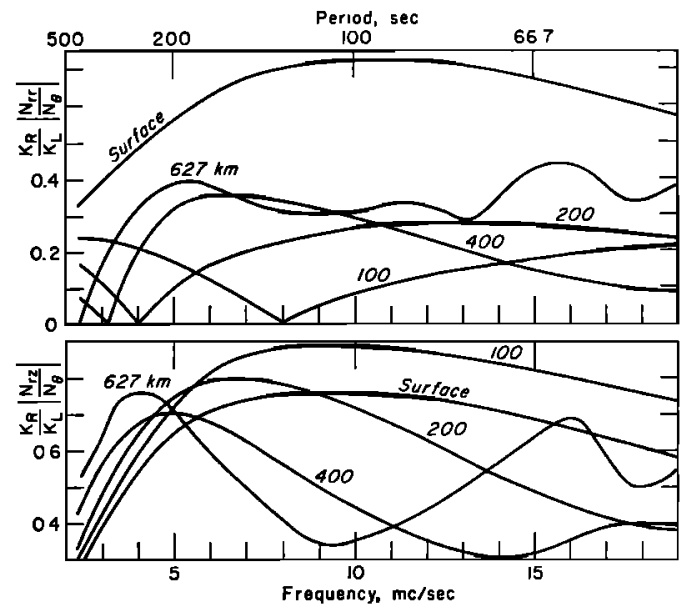

Fig. 16. Spectrum of singlet (Rayleigh/Love) excitation ratio for several values of the depth.
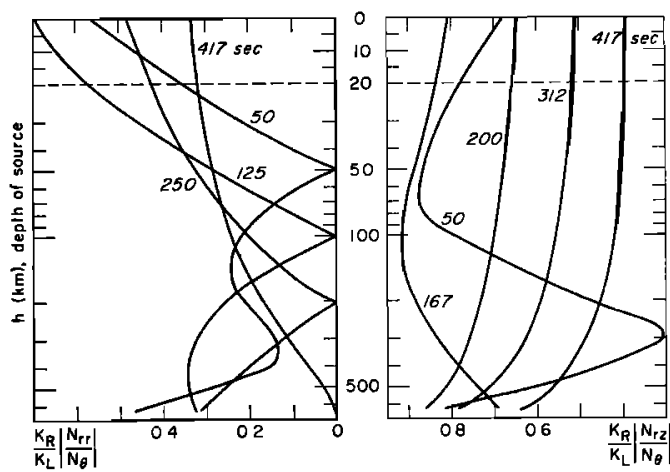

Fig. 17. Variation of singlet (Rayleigh/Love) excitation ratio with depth of source for various periods.

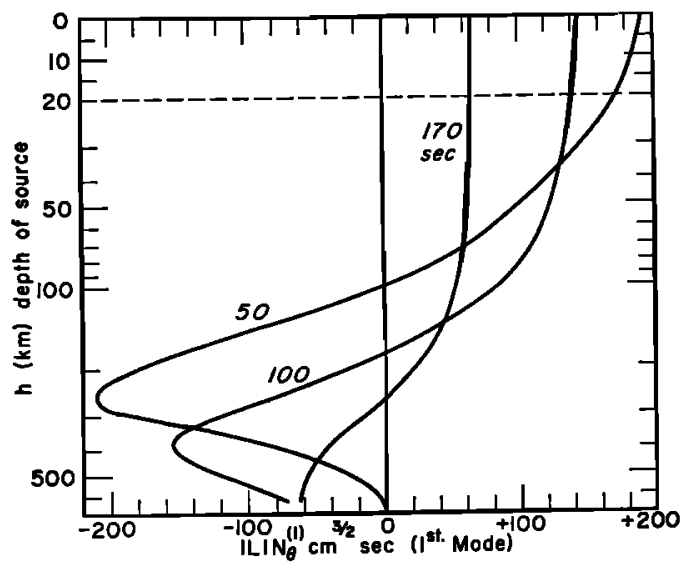

Fig. 18. Variation of the Love wave first-mode singlet transfer function with depth of source for various periods.

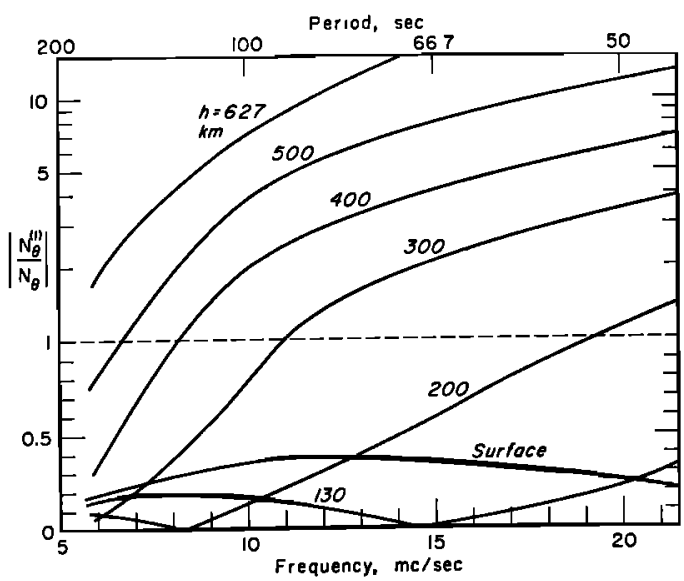

Fig. 19. Spectrum of (Love first-mode/Love fundamental-mode) excitation ratio for several values of the depth.

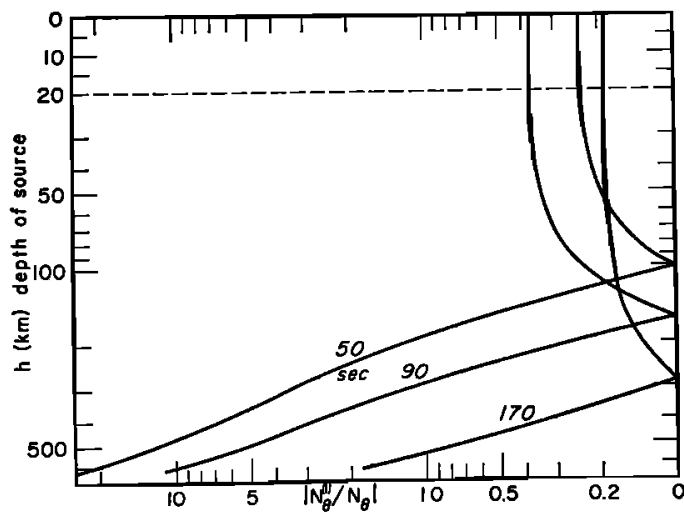

Fig. 20. Variations of (Love first-mode/Love fundamental-mode) excitation ratio for various periods.

The relative excitation functions of Rayleigh and Love waves are shown in Figure 16. The top figure represents the true spectral displacement ratio recorded at azimuth $\theta=45^{\circ}$ due to a horizontal force at depth. The variation with depth of $N_{\theta}^{(1)}(h, \omega)$, the Love wave first-mode singlet transfer function, is shown in Figure 18. The resemblance to $N_{r r}(h, \omega)$ (Figure 7 ) is apparent.

The relative excitation of the two Love modes is given in Figures 19 and 20. For a single force at depth, this ratio will depend neither on the orientation of the force nor on the azimuth of the station and will represent the true spectral displacement ratio. 
The functions $N_{\theta}, N_{r z}$, and $N_{r r}$ have the dimensions of $k_{n}{ }^{1 / 2} \mu^{-1}$. Since $k_{n}$ was expressed in $\mathrm{km}^{-1}$ and $\mu=\rho \beta^{2}$ in $\left(\mathrm{g} / \mathrm{cm}^{3}\right)(\mathrm{km} / \mathrm{sec})^{2}$, a conversion factor was applied to convert displacements to cgs units (Figures 2, 3, and 6 to 9).

\section{General Properties of the Radiation Patterns}

Symmetry relations. It is useful to look for symmetry with respect to the variable $\theta$ and the parameters $\lambda$ and $\delta$ in order to minimize the tabulation of the azimuthal distribution of the modulus $|\chi(\theta)|$ and the spatial phase arg $\chi(\theta)$. We denote by $m_{R}(\theta) e^{i \phi_{R}}$ and $m_{L}(\theta) e^{i \phi_{L}}$ the respective polar representations of the radiation patterns for Rayleigh and Love waves. It then follows directly from (24), (25), and Table 1 that

$\mathrm{U}(\theta+\pi ; \lambda, \delta, \omega, h)=\overline{\mathrm{U}}(\theta ; \lambda, \delta, \omega, h)$

for all couple and double-couple displacements. Equation 40 can also be written as

$$
\begin{gathered}
m_{R, L}(\theta+\pi)=m_{R, L}(\theta) \\
\phi_{R}(\theta+\pi)+\phi_{R}(\theta)=\phi_{L}(\theta+\pi)+\phi_{L}(\theta)=\pi / 2
\end{gathered}
$$

The relations given in (41) are due to $A k i$ [1964] and are useful for checking the calculations. It is assumed here that the source time dependence is a Dirac delta function. The antisymmetry between right-reverse and left-normal faultings yields

$$
\mathrm{U}(\lambda+\pi)=-\mathbf{U}(\lambda)
$$

Furthermore,
Auxiliary parameters and nodal lines. A simplification of the expressions for the displacement field is achieved upon the introduction of a parameter $\eta$ defined as

$$
\begin{aligned}
\cos \eta & =\cos \lambda\left(1-\sin ^{2} \lambda \sin ^{2} \delta\right)^{-1 / 2} \\
\sin \eta & =\sin \lambda \cos \delta\left(1-\sin ^{2} \lambda \sin ^{2} \delta\right)^{-1 / 2}
\end{aligned}
$$

The geometrical interpretation of $\eta$ is clear from Figure 1. We note also that $\phi=\eta-\pi / 2$ is the angle between the two dip directions of the plane of motion and the auxiliary plane. The dip angle of the auxiliary plane, $\delta^{\prime}$, is given by $\cos \delta^{\prime}=\sin \lambda \sin \delta$. From this relation, together with the definition given above, we find that

$$
\begin{aligned}
& \sin \eta=\cot \delta \cot \delta^{\prime}=\cos \phi \\
& \cos \lambda=|\cos p| \cos \eta \\
& \sin p=\sin \lambda \sin \delta
\end{aligned}
$$

With the aid of these angles we can transform (7) and (8) into

$$
\begin{aligned}
& U_{\theta}^{*}=|R| N_{\theta}(h) e^{23 \pi / 4}|\cos p| \sin (\theta-\eta) \\
& U_{r}^{*}=|R| N_{r z}(h) e^{-i \pi / 4}
\end{aligned}
$$

$$
\cdot[A(h)|\cos p| \cos (\theta-\eta)+i \sin p]
$$

A nodal line for Love waves exists at $\theta=\eta$, and its angular position is independent of the constants of the medium. The spatial phase is either $3 \pi / 4$ or $7 \pi / 4$. For Rayleigh waves a nodal line may exist only if $N_{r r}(h)=0$ or $p=0$. The phase will generally depend on the structure. Likewise we obtain for a couple source

$$
\begin{array}{r}
U_{\theta}{ }^{\circ}=|n||R| k_{L} N_{\theta}(h) e^{-i 3 \pi / 4}|\cos p| \sin (\theta-\eta)[-\sin \delta \sin \theta+i G(h) \cos \delta] \\
\begin{aligned}
& U_{r}{ }^{\circ}= \frac{1}{2}|n||R| k_{R} N_{r z}(h) e^{-i 3 \pi / 4}[|\cos p| \sin \delta\{A(h) \sin (2 \theta-\eta) \\
&+B(h) \sin \eta\} \\
&+2 i X(h) \sin (\theta+\zeta)]
\end{aligned}
\end{array}
$$

$$
\begin{gathered}
\mathrm{U}(-\delta)=-\overline{\mathbf{U}}(\delta) \\
U_{r}(\pi-\lambda, \pi-\theta)=U_{r}(\lambda, \theta) \\
U_{\theta}(\pi-\lambda, \pi-\theta)=-U_{\theta}(\lambda, \theta)
\end{gathered}
$$

which can be established from the nature of the coefficients in Table 1 . The foregoing relations show that it is sufficient to know the radiation pattern for $0 \leq \theta \leq \pi, 0 \leq \delta \leq \pi / 2$, and $0 \leq \lambda \leq \pi / 2$. The values for the supplementary angles can be obtained from these by reflections. where

$$
\begin{gathered}
X^{2}=\sin ^{2} \lambda\left[1-C(h) \cos ^{2} \delta\right]^{2} \\
+\cos ^{2} \lambda \cos ^{2} \delta(1-C)^{2} \\
\sin \zeta=[(1-C) /|X|] \cos \lambda \cos \delta \\
\cos \zeta=\left[\left(1-C \cos ^{2} \delta\right) / X\right] \sin \lambda
\end{gathered}
$$

and the value of $\zeta$ is determined by the algebraic signs of the two expressions. Thus for Love waves we always find at least one nodal line. 


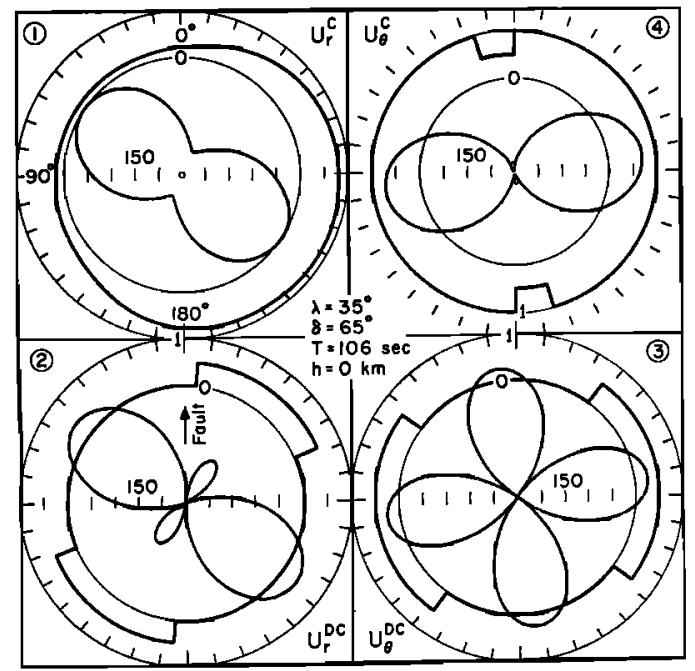

Fig. 21. Radiation pattern from two dipolar source models representing a left-reverse fault at zero depth for a spectral period of $106 \mathrm{sec}$ (numerical amplitude scale in all figures is for the reduced displacements).

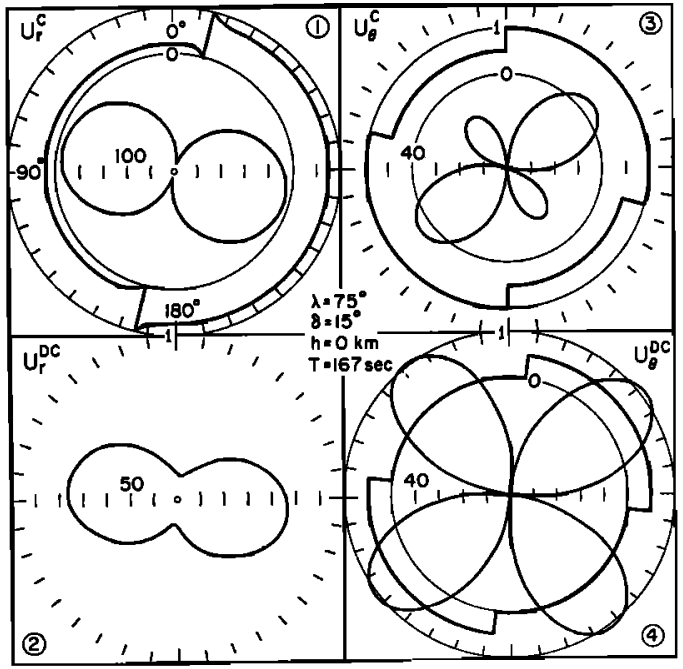

Fig. 22. Radiation pattern from two dipolar source models representing a left reverse fault at zero depth for a spectral period of $167 \mathrm{sec}$.

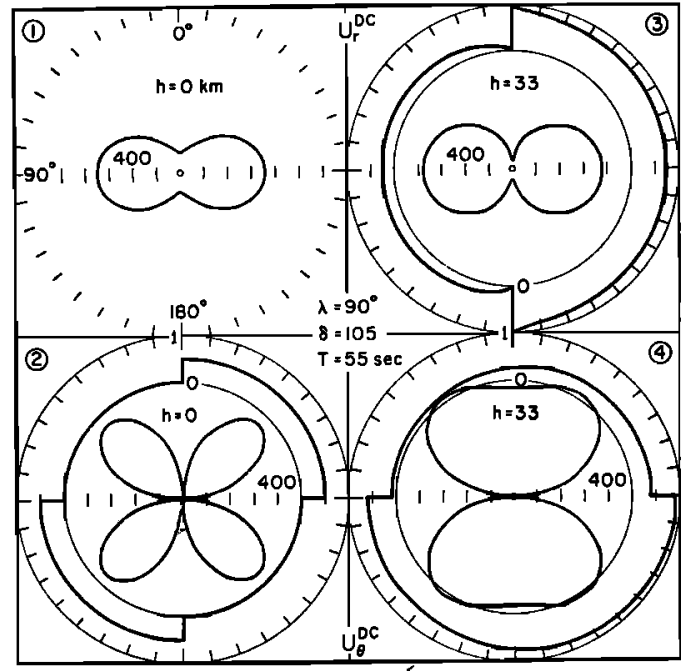

Fig. 23. Radiation pattern from a doublecouple source model representing a normal fault, at two distinct depths. Note change in Love wave radiation patterns as source is displaced from the free surface to base of crust.

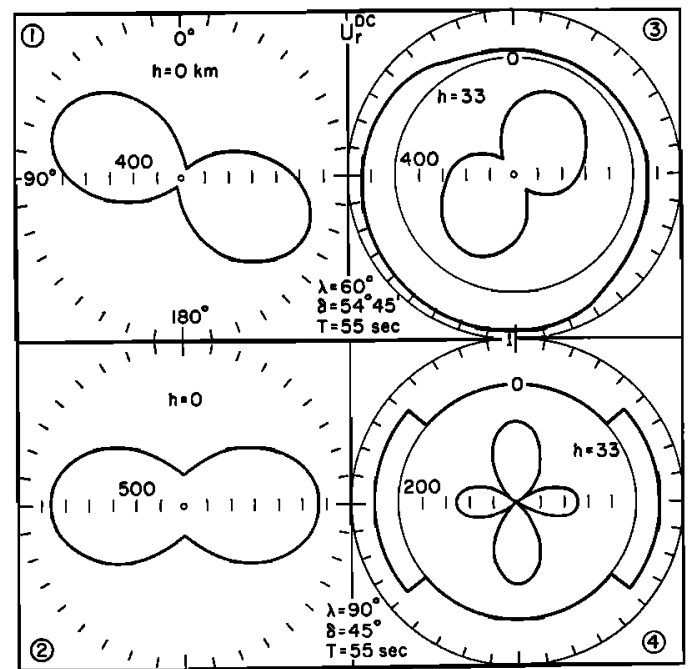

Fig. 24. Radiation pattern of Rayleigh waves from two double-couple source models representing left-reverse faults, each at two distinct depths. Note striking variations of radiation pattern in both cases as position of source changes from free surface to base of crust. 
The existence of the second node will depend on the dip angle. For higher modes $G(h)$ may vanish, and a clover-leaf pattern will be produced for some particular combinations of frequency and source depth, even for dip angles which differ from $\pi / 2$.

The Rayleigh wave pattern is more involved. There will be no nodes unless $\delta=0$ or $\lambda=\delta=\pi / 2$. The shape of the pattern will depend mainly on the relative magnitudes of $A, B$, and $C$. One may expect 'almost nodes' for frequency-depth combinations which render $C \gg A, B$ or $A \gg B, C$. The case $B \gg A, C$ will give an almost circular pattern. For the double-couple source we find
Unlike the previous case there will, in general, be no nodal lines for either Rayleigh or Love pattern, and the existence of 'almost nodes' will depend on the relative magnitude of the functions of the medium. The case $\delta=0$ will give a single node, and the case $\lambda=\pi / 2, B=0$ will give a quadrant pattern for both waves. A surface source will give a quadrant pattern for Love waves independent of the structure and the frequency. For Rayleigh waves there will be a node for $h=0$ only if $|\sin \eta| \leq\left(1-\sigma_{0}\right) /\left(1+\sigma_{0}\right)$. Expressing this condition in terms of the dip and slip angles we find that the dividing line for $\sigma_{0}=1 / 4$ is represented in the $(\lambda, \delta)$ plane by the curve

$$
\begin{array}{r}
U_{\theta}^{d c}=|n||R| k_{L} N_{\theta}(h) e^{-i 3 \pi / 4}[|\cos p| \sin \delta \cos (2 \theta-\eta)+i G(h)|\cos q| \sin (\theta-\Upsilon)] \\
\begin{array}{r}
U_{r}^{d c}=|n||R| k_{L} N_{r s}(h) e^{-i 3 \pi / 4}[|\cos p| \sin \delta\{A(h) \sin (2 \theta-\eta)+B(h) \sin \eta\} \\
-i C(h)|\cos q| \cos (\theta-\Upsilon)]
\end{array}
\end{array}
$$

where

$$
\cos \delta \cos \lambda=\cos \Upsilon|\cos q|
$$

$\cos \Upsilon=\cos \lambda \cos \delta\left(\cos ^{2} \lambda \cos ^{2} \delta\right.$

$$
\left.+\sin ^{2} \lambda \cos ^{2} 2 \delta\right)^{-1 / 2}
$$

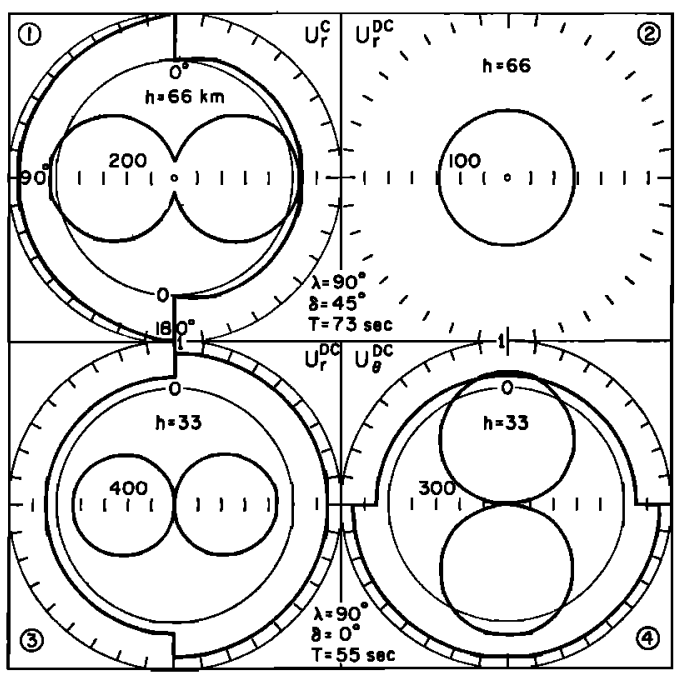

Fig. 25. Top: Radiation pattern of Rayleigh waves, couple versus double couple, for a reverse fault at depth of $66 \mathrm{~km}$. Bottom: Radiation pattern of Rayleigh waves, couple versus double couple, for a horizontal fault.

$$
\sin \lambda=3\left(25-16 \sin ^{2} \delta\right)^{-1 / 2}
$$

Dependence of patterns on the source elements. The modulus $|\chi(\theta)|$ describes in the general case an obliquely symmetric, closed curve of the sixth order, otherwise known as the hypertrochoid. Its shape is governed by the coefficients $d_{i}$ (Table 1) which in turn are composite func-

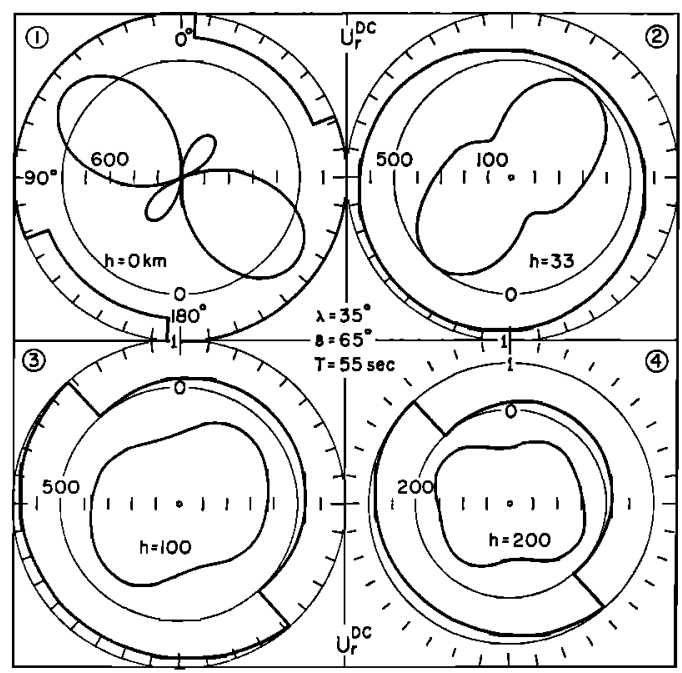

Fig. 26. The effect of source depth on the Rayleigh wave radiation pattern from a left-reverse fault with a double-couple force system. Note radical change of radiation pattern as source is displaced from the free surface to base of crust. 


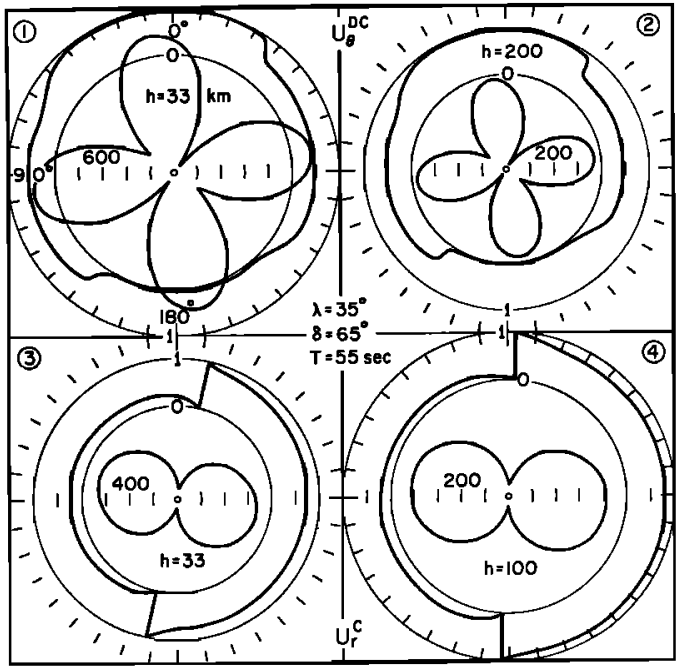

Fig. 27. Top: The effect of source depth on radiation pattern of Love waves from a leftreverse fault (double couple). Bottom: The effect of source depth on radiation pattern of Rayleigh waves from a left-reverse fault (single couple).

tions of the source constants $(\lambda, \delta, h)$, the period $T$, and the physical constants of the earth model. To study the effects of the variation of these parameters on the radiation pattern we have plotted some typical examples. (Figures 21 to 29). Azimuthal variation of amplitude is at the center of the figure. The peripheral curve shows azimuthal variation of the corresponding phase in a unit ring [Brune, 1961; Haskell, 1963]. The amplitude plotted in these figures is the reduced amplitude $\left|U^{*}\right|$, which is a normalized entity of dimensions $\mathrm{cm}^{1 / 2} /$ dyne. It is related to the true spectral amplitude $|U|$ through the equation

$$
|U|=\frac{10^{-19}|R(\omega)||n|}{(2 \pi r)^{1 / 2}}\left|U^{*}\right| e^{-\gamma(\omega) r}
$$

$|R(\omega)|$ is the magnitude of the force in cgs units, $r$ is the epicentral distance in $\mathrm{km}$, and $\gamma(\omega)$ is the absorption coefficient in $\mathrm{km}^{-1}$. The analytic expressions of the patterns shown in Figures 21 to 29 are given in Table 3 . In choosing the various examples it was intended to emphasize strong variations of patterns with respect to depth of source, frequency, mode, and force configuration. Note the stationary behavior of the phases at azimuths where the amplitude is maximum and its rapid variation at azimuths where the amplitude is relatively minimum. This develops into an extreme case at which a jump of $\pi$ radians occurs at the nodal lines. The phases in all figures vary between zero and $2 \pi$ radians. The additional constant phase for each case is given in Table 3 .

\section{Discussion and Conchusions}

Previous endeavors in this field were restricted either to simpler types of mediums or to sources

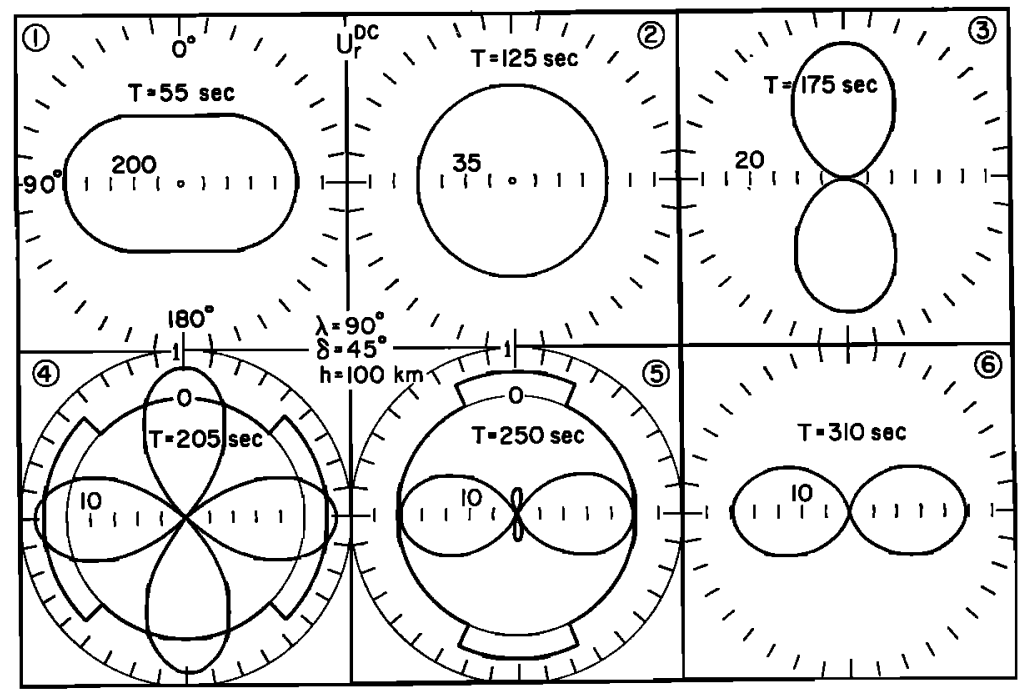

Fig. 28. The effect of the source frequency on the radiation pattern of Rayleigh waves from a reverse fault (double couple) at depth of $100 \mathrm{~km}$. 


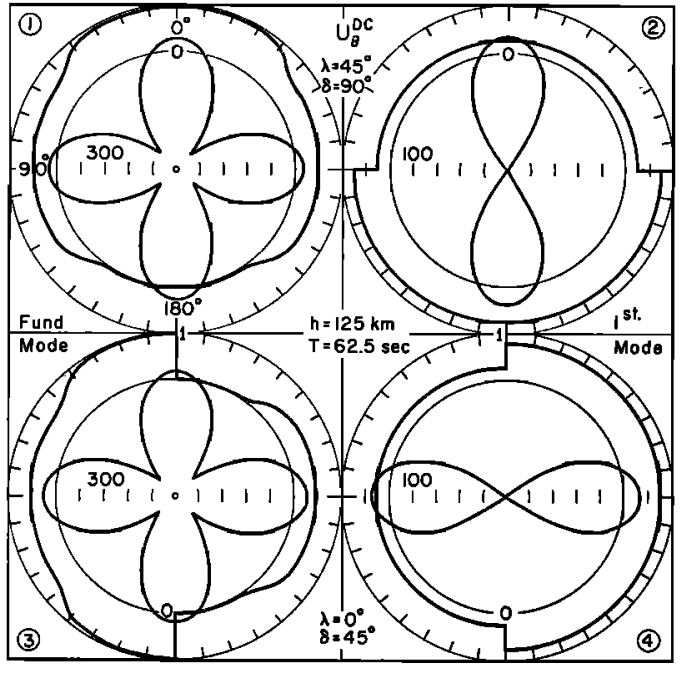

Fig. 29. Dependence of radiation pattern of Love waves upon the displacement mode for two different sources. Top: Vertical fault (double couple). Bottom: Strike-slip fault (double couple).

of lesser complexity, or both. In the present study we have made an effort to overcome these unnecessary limitations. As usual, this requires increasing the number of the participating physical parameters, such as the depth of source, frequency, dip and slip angles, and finally the constants of the elastic medium.

Radiation patterns were calculated for periods less than $350 \mathrm{sec}$, since we believed that a sphericity correction for longer periods was imperative. It would be advantageous to recalculate the transfer functions of the medium for a multilayered spherical earth whenever a suitable computer program becomes available. It would also prove useful to study the sensitivity of the transfer functions to variations of the constants of the medium. The case of an oceanic path is of particular interest.

The method given here can be extended to encompass a wider class of sources. Multipolar sources may be obtained by applying a differential operator of a suitable degree to the singlet field. A horizontal extension of the source introduces a frequency-dependent asymmetry factor into the displacement spectrums, and a vertical extension will necessitate an integration of the displacements with depth [Ben-Menahem, 1961]. Other source representations, such as proposed by Knopoff and Gilbert [1960] and Aki [1964], can also be treated by our method.

The radiation pattern is an important signature of the seismic source. It can and should be studied more intensively, therefore, in order to
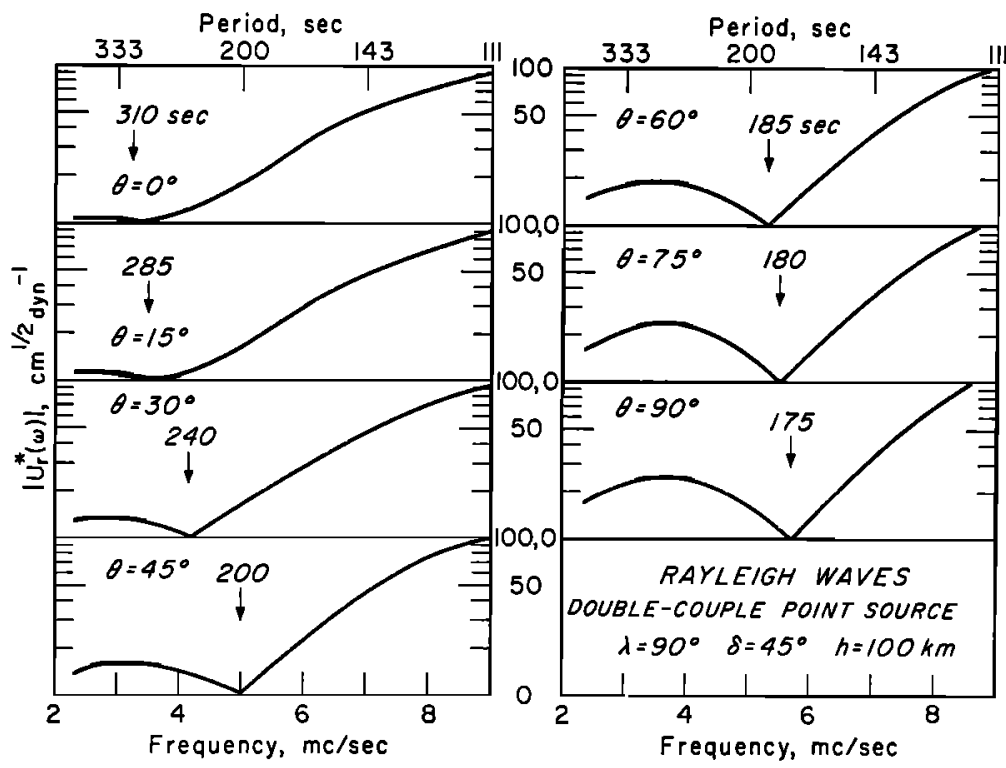

Fig. 30. Variation of the reduced amplitude spectrum with azimuth (from strike of fault) for same source as in Figure 28. Note progression of spectral node toward shorter periods as azimuth changes from $0^{\circ}$ to $90^{\circ}$. 
TABLE 3. Radiation Pattern Coefficients of the Reduced Displacements

$U^{*}=U_{0} e^{-i \phi_{0}}\left[a_{0}+b_{0} \sin \left(2 \theta-\theta_{0}\right)+i b_{1} \sin \left(\theta+\theta_{1}\right)\right]$ Shown in Figures 21 to 29

The angle $\phi_{0}$ has been suppressed in the phase-ring diagrams of these figures.

\begin{tabular}{|c|c|c|c|c|c|c|c|c|c|}
\hline Figure & Wave* & Source $†$ & $\begin{array}{c}U_{0} \\
\mathrm{~cm}^{1 / 2} / \mathrm{dyne}\end{array}$ & $a_{0}$ & $b_{0}$ & $b_{1}$ & $\begin{array}{l}\theta_{0} \\
\operatorname{deg}\end{array}$ & $\begin{array}{c}\theta_{1}, \\
\operatorname{deg}\end{array}$ & $\begin{array}{l}\phi_{0} \\
\mathrm{rad}\end{array}$ \\
\hline $\begin{array}{r}21(1) \\
(2) \\
(3) \\
(4)\end{array}$ & $\begin{array}{l}R \\
R \\
L \\
L\end{array}$ & $\begin{array}{l}C \\
D C \\
D C \\
C\end{array}$ & $\begin{array}{l}148.0 \\
2960 \\
418.0 \\
214.0\end{array}$ & $\begin{array}{l}.46 \\
.46 \\
0 \\
.96\end{array}$ & $\begin{array}{r}1 \\
1 \\
1 \\
-1\end{array}$ & $\begin{array}{c}2.08 \\
0 \\
0 \\
0\end{array}$ & $\begin{array}{r}16.4 \\
16.4 \\
-73.6 \\
-73.6\end{array}$ & $\begin{array}{l}31.1 \\
0 \\
0 \\
0\end{array}$ & $\begin{array}{r}3 \pi / 4 \\
3 \pi / 4 \\
3 \pi / 4 \\
-\pi / 4\end{array}$ \\
\hline $\begin{array}{r}22(1) \\
(2) \\
(3) \\
(4)\end{array}$ & $\begin{array}{l}R \\
R \\
L \\
L\end{array}$ & $\begin{array}{l}C \\
D C \\
C \\
D C\end{array}$ & $\begin{array}{l}22.7 \\
45.4 \\
35.6 \\
71.3\end{array}$ & $\begin{array}{c}1.57 \\
1.57 \\
.27 \\
0\end{array}$ & $\begin{array}{r}1 \\
1 \\
-1 \\
1\end{array}$ & $\begin{array}{c}10.20 \\
0 \\
0 \\
0\end{array}$ & $\begin{array}{r}74.5 \\
74.5 \\
-15.5 \\
-15.5\end{array}$ & $\begin{array}{l}14.5 \\
0 \\
0 \\
0\end{array}$ & $\begin{array}{r}3 \pi / 4 \\
3 \pi / 4 \\
-\pi / 4 \\
3 \pi / 4\end{array}$ \\
\hline $\begin{array}{r}23(1) \\
(2) \\
(3) \\
(4)\end{array}$ & $\begin{array}{l}R \\
L \\
R \\
L\end{array}$ & $\begin{array}{l}D C \\
D C \\
D C \\
D C\end{array}$ & $\begin{array}{r}270 \\
447 \\
74.0 \\
375.0\end{array}$ & $\begin{array}{c}1.6 \\
0 \\
.15 \\
0\end{array}$ & $\begin{array}{r}-1 \\
1 \\
1 \\
1\end{array}$ & $\begin{array}{c}0 \\
0 \\
10.00 \\
1.24\end{array}$ & $\begin{array}{r}-90 \\
0 \\
-90 \\
0\end{array}$ & $\begin{array}{c}0 \\
0 \\
0 \\
90\end{array}$ & $\begin{array}{r}-\pi / 4 \\
-\pi / 4 \\
3 \pi / 4 \\
-\pi / 4\end{array}$ \\
\hline $\begin{array}{r}24(1) \\
(2) \\
(3) \\
(4)\end{array}$ & $\begin{array}{l}R \\
R \\
R \\
R\end{array}$ & $\begin{array}{l}D C \\
D C \\
D C \\
D C\end{array}$ & $\begin{array}{l}620.0 \\
537.0 \\
173.1 \\
150.0\end{array}$ & $\begin{array}{r}1.15 \\
1.63 \\
.10 \\
.14\end{array}$ & $\begin{array}{r}1 \\
-1 \\
-1 \\
1\end{array}$ & $\begin{array}{c}0 \\
0 \\
-2.04 \\
0\end{array}$ & $\begin{array}{r}45 \\
-90 \\
45 \\
-90\end{array}$ & $\begin{array}{c}0 \\
0 \\
-45 \\
0\end{array}$ & $\begin{array}{r}3 \pi / 4 \\
3 \pi / 4 \\
-\pi / 4 \\
-\pi / 4\end{array}$ \\
\hline $\begin{array}{r}25(1) \\
(2) \\
(3) \\
(4)\end{array}$ & $\begin{array}{l}R \\
R \\
R \\
L\end{array}$ & $\begin{array}{l}C \\
D C \\
D C \\
D C\end{array}$ & $\begin{array}{r}71.2 \\
142.4 \\
866.0 \\
563.0\end{array}$ & $\begin{array}{l}1 \\
1 \\
\quad 0 \\
0\end{array}$ & $\begin{array}{l}0 \\
0 \\
0 \\
0\end{array}$ & $\begin{array}{c}-7.32 \\
0 \\
1 \\
1\end{array}$ & $\begin{array}{l}0 \\
0 \\
0 \\
0\end{array}$ & $\begin{array}{c}0 \\
0 \\
0 \\
90\end{array}$ & $\begin{array}{r}-\pi / 4 \\
-\pi / 4 \\
-\pi / 4 \\
3 \pi / 4\end{array}$ \\
\hline $\begin{array}{r}26(1) \\
(2) \\
(3) \\
(4)\end{array}$ & $\begin{array}{l}R \\
R \\
R \\
R\end{array}$ & $\begin{array}{l}D C \\
D C \\
D C \\
D C\end{array}$ & $\begin{array}{r}831.2 \\
232.0 \\
162.1 \\
85.5\end{array}$ & $\begin{array}{r}.46 \\
.04 \\
1.04 \\
.91\end{array}$ & $\begin{array}{r}1 \\
-1 \\
1 \\
1\end{array}$ & $\begin{array}{c}0 \\
1.89 \\
2.53 \\
1.86\end{array}$ & $\begin{array}{l}16.4 \\
16.4 \\
16.4 \\
16.4\end{array}$ & $\begin{array}{c}0 \\
136.8 \\
136.8 \\
136.8\end{array}$ & $\begin{array}{l}3 \pi / 4 \\
-\pi / 4 \\
-\pi / 4 \\
-\pi / 4\end{array}$ \\
\hline $\begin{array}{r}27(1) \\
(2) \\
(3) \\
(4)\end{array}$ & $\begin{array}{l}L \\
L \\
R \\
R\end{array}$ & $\begin{array}{l}D C \\
D C \\
C \\
C\end{array}$ & $\begin{array}{r}1161.0 \\
375.3 \\
116.0 \\
81.0\end{array}$ & $\begin{array}{c}0 \\
0 \\
.04 \\
1.04\end{array}$ & $\begin{array}{r}1 \\
1 \\
-1 \\
1\end{array}$ & $\begin{array}{r}-.23 \\
-.22 \\
5.79 \\
4.89\end{array}$ & $\begin{array}{r}-73.6 \\
-73.6 \\
16.4 \\
16.4\end{array}$ & $\begin{array}{r}46.8 \\
46.8 \\
12.7 \\
1.2\end{array}$ & $\begin{array}{r}3 \pi / 4 \\
3 \pi / 4 \\
-\pi / 4 \\
-\pi / 4\end{array}$ \\
\hline $\begin{array}{r}28(1) \\
(2) \\
(3) \\
(4) \\
(5) \\
(6)\end{array}$ & $\begin{array}{l}R \\
R \\
R \\
R \\
R \\
R\end{array}$ & $\begin{array}{l}D C \\
D C \\
D C \\
D C \\
D C \\
D C\end{array}$ & $\begin{array}{r}104.6 \\
71.2 \\
28.0 \\
16.0 \\
57.5 \\
11.5\end{array}$ & $\begin{array}{l}3.68 \\
1 \\
1 \\
0 \\
0.62 \\
1\end{array}$ & $\begin{array}{r}1 \\
0 \\
1 \\
1 \\
-1 \\
-1\end{array}$ & $\begin{array}{l}0 \\
0 \\
0 \\
0 \\
0 \\
0\end{array}$ & $\begin{array}{r}90 \\
0 \\
-90 \\
-90 \\
-90 \\
-90\end{array}$ & $\begin{array}{l}0 \\
0 \\
0 \\
0 \\
0 \\
0\end{array}$ & $\begin{array}{r}-\pi / 4 \\
-\pi / 4 \\
-\pi / 4 \\
-\pi / 4 \\
3 \pi / 4 \\
3 \pi / 4\end{array}$ \\
\hline $\begin{array}{r}29(1) \\
(2) \\
(3) \\
(4)\end{array}$ & $\begin{array}{l}L \\
L^{(1)} \\
L^{(1)} \\
L^{(1)}\end{array}$ & $\begin{array}{l}D C \\
D C \\
D C \\
D C\end{array}$ & $\begin{array}{l}538.0 \\
142.8 \\
538.0 \\
142.8\end{array}$ & $\begin{array}{l}0 \\
0 \\
0 \\
0\end{array}$ & $\begin{array}{l}1 \\
0 \\
1 \\
0\end{array}$ & $\begin{array}{l}-.25 \\
\frac{1}{1} .25\end{array}$ & $\begin{array}{r}-90 \\
0 \\
-90 \\
0\end{array}$ & $\begin{array}{c}90 \\
90 \\
0 \\
0\end{array}$ & $\begin{array}{r}3 \pi / 4 \\
-\pi / 4 \\
3 \pi / 4 \\
-\pi / 4\end{array}$ \\
\hline
\end{tabular}

${ }^{*} L=$ Love, $R=$ Rayleigh.

$\dagger C=$ couple, $D C=$ double couple.

eliminate the guesswork inherent in the firstmotion method and to put seismic source studies on a firm physical basis.

Acknowledgments. This research was supported by contract AF-49(638)-1337 of the Air Force Office of Scientific Research as part of the Advanced Research Projects Agency project Vela.
We wish to thank Keiiti Aki for valuable discussions during the early stages of this work.

\section{ReFERENCES}

Aki, K., Study of Love and Rayleigh waves from earthquakes with fault plane solutions or with known faultings, Bull. Seismol. Soc. Am., in press, 1964. 
Ben-Menahem, A., Radiation of seismic surface waves from finite moving sources, Bull. Seismol. Soc. Am., 51, 401-435, 1961.

Ben-Menahem, A., and M. Nafi Toksöz, Source mechanism from spectra of long-period seismic surface waves, 2, The Kamchatka earthquake of November 4, 1952, J. Geophys. Res., 68, 52075222, 1963.

Brune, J. N., Radiation pattern of Rayleigh waves from the southeast Alaska earthquake of July 10, 1958, Publ. Dominion Obs., Ottawa, 24(10), 1-11, 1961.

Harkrider, D. G., Surface waves in multilayered elastic media, 1. Rayleigh and Love waves from buried sources in a multilayered elastic halfspace, Bull. Seismol. Soc. Am., in press, 1964a. 2. Rayleigh and Love wave seismograms and spectra from buried sources in a multilayered elastic half-space, in preparation, $1964 b$.

Haskell, N. A., The dispersion of surface waves in multilayered media, Bull. Seismol. Soc. Am., 43, 17-34, 1953.

Haskell, N. A., Radiation pattern of Rayleigh waves from a fault of arbitrary dip and direction of motion in a homogeneous medium, Bull. Seismol. Sac. Am., 53, 619-642, 1963.

Knopoff, L, and F. Gilbert, First motions from seismic sources, Bull. Seismol. Soc. Am., 50, 117$134,1960$.

Yanovskaya, T. B., On the determination of the dynamic parameters of the focus hypocenter of an earthquake from records of surface waves, Izv. Akad. Nauk SSSR, Ser. Geofiz., 209-301, 1958.

(Manuscript received February 21, 1964.) 